\title{
Robust multiperiod portfolio management in the presence of transaction costs
}

\author{
Dimitris Bertsimas $^{\mathrm{a}}$, Dessislava Pachamanova ${ }^{\mathrm{b}, *}$ \\ ${ }^{a}$ Sloan School of Management and Operations Research Center, Massachusetts Institute of Technology, Building E53-363, \\ Cambridge, MA 02142-1347, USA \\ ${ }^{\mathrm{b}}$ Division of Mathematics and Sciences, Babson College, Forest Street, Babson Park, MA 02457, USA
}

Available online 23 March 2006

\begin{abstract}
We study the viability of different robust optimization approaches to multiperiod portfolio selection. Robust optimization models treat future asset returns as uncertain coefficients in an optimization problem, and map the level of risk aversion of the investor to the level of tolerance of the total error in asset return forecasts. We suggest robust optimization formulations of the multiperiod portfolio optimization problem that are linear and computationally efficient. The linearity of the optimization problems is an advantage when complex additional requirements need to be imposed on the portfolio structure, e.g., limitations on positions in certain assets or tax constraints. We compare the performance of our robust formulations to the performance of the traditional single period mean-variance formulation frequently employed in the financial industry.
\end{abstract}

(C) 2006 Elsevier Ltd. All rights reserved.

Keywords: Robust optimization; Multiperiod portfolio management

\section{Literature overview and positioning}

The first systematic approach to the problem of asset allocation under uncertainty is attributed to Markowitz [1]. Markowitz's seminal paper addressed the important issue of tradeoff between risk and return. It looked at portfolio selection as an optimization problem in which an asset mix is chosen so that the portfolio variance is minimal for any given level of expected return, and simultaneously, the expected return is maximal for any given level of portfolio variance. The mean-variance formulation can be written as follows:

$$
\max \left\{\check{\mathbf{r}}^{\prime} \mathbf{x}-\lambda \mathbf{x}^{\prime} \Sigma \mathbf{x} \mid \mathbf{x}^{\prime} \mathbf{e}=1\right\},
$$

where $\mathbf{x} \in \mathfrak{R}^{n}$ is the vector of asset weights, $\breve{\mathbf{r}} \in \mathfrak{R}^{n}$ and $\Sigma \in \mathfrak{R}^{n \times n}$ are the vector of expected values and the covariances matrix of the asset returns, respectively, $\mathbf{e}$ is a vector of ones, and $\lambda$ is a penalty parameter associated with the investor's risk preferences.

A fundamental problem with the original Markowitz analysis and the generalized mean-risk models that have sprung from the mean-variance approach is their single period nature. Inappropriate choice of the length of the time horizon can lead to suboptimal investment decisions. Markowitz [2, Chapters X-XIII] discussed long-term investment planning

\footnotetext{
* Corresponding author.

E-mail addresses: dbertsim@mit.edu (D. Bertsimas), dpachamanova@babson.edu (D. Pachamanova).
} 
in the context of a utility function based on consumption, and placed the problem in the realm of dynamic programming. Analytic solutions to the continuous version of the investor's optimization problem for special types of utility functions and asset price processes have been subsequently studied [3-6]. However, closed-form solutions of this kind can be derived only under strong assumptions on the investor's behavior and the structure of the asset price process, and do not generalize easily when market frictions, e.g., transaction costs, are included.

Recent advances in computer technology have reduced the significance of the ability to solve the multiperiod portfolio problem in closed form, and have made discrete time portfolio optimization models more tractable. Techniques from approximate dynamic programming have been successfully employed for efficient optimal policy computations: for example, Chryssikou [7] uses approximate dynamic programming algorithms to provide a near-optimal dynamic trading strategy for special types of utility functions when a closed form solution to the discrete-time multiperiod problem with quadratic transaction costs is not attainable. A significant amount of research has been carried out also in the field of stochastic programming applications to portfolio optimization [8-11]. The main idea of the stochastic programming approach is to represent future realizations of returns by a scenario tree, and find portfolio weights at each point in time that maximize the expected value of the returns over the whole time horizon minus some measure of risk. Although this analysis is very flexible from a modeling standpoint, its computational complexity increases exponentially with the number of time periods.

\subsection{The robust optimization approach to portfolio management}

Robust optimization has emerged as a leading methodology for addressing uncertainty in optimization problems. Suppose we are given a linear optimization problem

$$
\max \left\{\mathbf{c}^{\prime} \mathbf{x} \mid \tilde{\mathbf{A}} \mathbf{x} \leqslant \mathbf{b}, \mathbf{x} \in P^{x}\right\}
$$

where $\mathbf{x} \in \mathfrak{R}^{n \times 1}$ is a vector of variables, $\tilde{\mathbf{A}} \in \mathfrak{R}^{m \times n}$ is a matrix of uncertain coefficients, and $\mathbf{c} \in \mathfrak{R}^{n \times 1}$ is a vector of objective function coefficients, and $P^{x}$ is a given set representing the constraints involving only certain coefficients. The robust counterpart of (2) is

$$
\max \left\{\mathbf{c}^{\prime} \mathbf{x} \mid \tilde{\mathbf{A}} \mathbf{x} \leqslant \mathbf{b}, \mathbf{x} \in P^{x}, \forall \tilde{\mathbf{A}} \in \mathscr{U}\right\}
$$

For arbitrary uncertainty sets $\mathscr{U}$ this problem is a semi-infinite optimization problem. However, for certain types of uncertainty sets (in particular, ellipsoids), the problem can be formulated as an explicit convex program with certain coefficients [12,13]. Its optimal solution $\mathbf{x}$ is robust, i.e., it satisfies the constraints of the problem for any $\tilde{\mathbf{A}} \in \mathscr{U}$, and in particular, it satisfies the constraints for the worst-case value of $\tilde{\mathbf{A}}$ in $\mathscr{U}$.

Ben-Tal et al. [14] were the first to suggest using robust optimization to deal with the curse of dimensionality in multiperiod portfolio optimization problems. Their problem formulation can be viewed as an extension of the Certainty Equivalent Controller (CEC) procedure from dynamic programming [15]. The CEC represents a deterministic approach to uncertainty - at each stage, it applies the policy that is optimal when all uncertain quantities are fixed at their expected values. An important disadvantage of the CEC approach is that risk is not factored in. Ben-Tal et al. [14] incorporate risk by allowing future asset returns to vary in ellipsoidal sets whose size is determined by the user and depends on his aversion to uncertainty. The robust counterpart of the multiperiod portfolio optimization problem can then be formulated as a second order cone problem (SOCP). Although this method appears simplified, there are several reasons it may be of practical interest. First, it has been shown that in the single period case, restricting the uncertain returns to vary jointly in ellipsoidal sets determined by the inverse of the returns' covariance matrix results in a robust counterpart of the single period portfolio optimization problem that is reminiscent of the Markowitz original mean-risk framework: the robust counterpart maximizes the expected return of the portfolio minus a penalty coefficient times the standard deviation of the portfolio [16]. A generalization of this result to the multiperiod portfolio optimization case appears to be a natural extension. Second, the computational results in [14] indicate that Ben-Tal et al.'s robust optimization formulation for ellipsoidal uncertainty sets outperforms stochastic programming algorithms both in terms of efficiency and in terms of optimal strategy selection. 


\subsection{Structure and contributions of this paper}

Ben-Tal et al.'s [14] idea of using robust optimization to formulate the multiperiod portfolio optimization problem shows excellent promise as a computationally efficient alternative to existing methods for multiperiod portfolio management. However, some issues merit further research. First, Ben-Tal et al.'s [14] formulation is convex, and thus, computationally tractable. However, it is nonlinear. A linear formulation may be preferable when, in order to incorporate complex tax constraints and portfolio structure requirements, one may need to resort to integer programming (see, for example, Bertsimas et al. [17]). In such cases, there is an advantage to starting with a linear framework, as nonlinear mixed integer programming software is not as well developed as linear mixed integer programming software. Robust mixed integer programming models are studied in Bertsimas and Sim [18]. Second, it is interesting to test not only whether the multiperiod robust optimization approach outperforms multiperiod stochastic programming algorithms, but also whether it is better than existing single period portfolio optimization alternatives that are used in practice.

In this paper, we build on Ben-Tal et al.'s [14] approach; however, we work with polyhedral (as opposed to ellipsoidal) uncertainty sets for future returns based on Bertsimas et al. [19]. Bertsimas et al. [19] study the robust counterparts of linear optimization problems when the total distance (according to a pre-specified norm) between the realized uncertain coefficients and their nominal values is restricted to be less than a "robustness budget" $\Delta$. They define a norm called the $D$-norm that includes the polyhedral norms $L_{1}$ and the $L_{\infty}$ as special cases, and demonstrate that for $d=\sqrt{n}$, where $n$ is the dimension of the vector of uncertain returns, the $D$-norm approaches the $L_{2}$ norm. Thus, the uncertainty sets defined for $d=\sqrt{n}$ approximate ellipsoids. We propose new robust formulations for the multiperiod portfolio optimization problem, and study the computational performance of the proposed models in numerous simulations, benchmarking it against the performance of a classical method of portfolio allocation used extensively in industry: single period mean-variance optimization.

The structure of this paper is as follows: Section 2 defines the problem of multiperiod portfolio management with transaction costs. Section 3 presents different robust formulations for the multiperiod portfolio optimization problem. Section 4 contains computational results on the performance of two of the suggested multiperiod robust portfolio optimization methods relative to single period optimization techniques. Section 5 concludes with a summary of findings.

\subsection{Notation}

The following notation will be used in this paper:

- Boldface denotes vectors and matrices. Upper case letters (e.g., A) are used for matrices, and lower case letters (e.g., a) are used for vectors. $\mathbf{a}_{i}$ stands for the $i$ th row of a matrix $\mathbf{A}$;

- Tilde (e.g., $\tilde{a}$ ) denotes uncertain coefficients;

- Check (e.g., $\check{a}$ ) denotes the expected value of $a$;

- Overline and underline (e.g., $\bar{a}$ and $\underline{a}$ ) denote upper and lower bound on $a$, respectively;

- $\||\mathbf{x}|\|_{d}$ denotes the $D$-norm of a vector $\mathbf{x} \in \mathfrak{R}^{n}$, as introduced in Bertsimas et al. [19], and equals $\max _{\{S \cup\{t\}|S \subseteq N,| S \mid \leqslant\lfloor d\rfloor, t \in N \backslash S\}}\left\{\sum_{j \in S}\left|x_{j}\right|+(d-\lfloor d\rfloor)\left|x_{t}\right|\right\}$.

\section{The multiperiod portfolio optimization problem}

The multiperiod portfolio management problem with linear transaction costs can be formulated as follows: there are $M$ risky assets, one riskless asset (asset 0 ), and $N$ trading periods, $t=0, \ldots, N-1$. At time period $N$, an investor collects his final wealth $W_{N}$. His goal is to manage the portfolio of assets in a manner that maximizes his expected utility of final wealth $U\left(W_{N}\right)$.

The investor's dollar holdings at the beginning of time period $t, t=0,1, \ldots, N$, are $x_{t}^{m}, m=0,1, \ldots, M$. If he sells an amount $u_{t}^{m}$ or buys an amount $v_{t}^{m}$ of stock $m$ at time $t$, he incurs transaction costs of $c_{\text {sell }} u_{t}^{m}$ and $c_{\text {buy }} v_{t}^{m}$, respectively. Proceeds from the sales are added to, and expenses from the purchases are subtracted from, the cash account, asset 0 . At time $t+1$, the investor's holdings are updated according to the realized returns over $(t, t+1]$. Let the (uncertain) return of stock $m$ over time period $(t, t+1]$ be $\tilde{r}_{t}^{m}$. For simplicity, we will assume that the single period returns of the riskless asset, $r_{t}^{0}$, are fixed, although this framework allows for modeling uncertainty in $r_{t}^{0}$. 
The dynamics of the investor's holdings are then given by the equations

$$
\begin{aligned}
& x_{t}^{m}=\left(1+r_{t-1}^{m}\right)\left(x_{t-1}^{m}-u_{t-1}^{m}+v_{t-1}^{m}\right), \quad t=1, \ldots, N, \quad m=1, \ldots, M, \\
& x_{t}^{0}=\left(1+r_{t-1}^{0}\right)\left(x_{t-1}^{0}+\sum_{m=1}^{M}\left(1-c_{\text {sell }}\right) u_{t-1}^{m}-\sum_{m=1}^{M}\left(1+c_{\text {buy }}\right) v_{t-1}^{m}\right), \quad t=1, \ldots, N .
\end{aligned}
$$

If the investor could foresee the realizations of the uncertain returns $\tilde{r}_{t}^{m}, t=0, \ldots, N-1, m=1, \ldots, M$, his optimal strategy would be given by the optimal solution to the following optimization problem:

$$
\begin{aligned}
\left(\mathscr{P}^{r}\right) \max & U\left(\sum_{m=0}^{M} x_{N}^{m}\right) \\
\text { s.t. } \quad & x_{t}^{m}=\left(1+\tilde{r}_{t-1}^{m}\right)\left(x_{t-1}^{m}-u_{t-1}^{m}+v_{t-1}^{m}\right), \quad t=1, \ldots, N, \quad m=1, \ldots, M, \\
& x_{t}^{0}=\left(1+r_{t-1}^{0}\right)\left(x_{t-1}^{0}+\sum_{m=1}^{M}\left(1-c_{\text {sell }}\right) u_{t-1}^{m}-\sum_{m=1}^{M}\left(1+c_{\text {buy }}\right) v_{t-1}^{m}\right), \quad t=1, \ldots, N, \\
& x_{t}^{m} \geqslant 0, \quad t=1, \ldots, N, \quad m=0, \ldots, M, \\
& u_{t}^{m} \geqslant 0, \quad v_{t}^{m} \geqslant 0, \quad t=1, \ldots, N, \quad m=1, \ldots, M .
\end{aligned}
$$

Here we have imposed nonnegativity constraints on the investor's holdings at each time period, which is equivalent to not allowing for borrowing or short sales.

In reality, of course, future returns are not known at time 0 . In practice, the investor has to treat the portfolio optimization problem as a rolling horizon problem, i.e., he has to act upon information available at time $t$, and rebalance his portfolio at time $t+1$ after obtaining additional information over time period $(t, t+1]$. We will adopt the rolling horizon philosophy in the application of robust formulations for multiperiod portfolio optimization. We will assume that at each time period, the investor takes only the first step of the optimal allocation strategy computed with information up to that time period, i.e., that he solves consecutive multiperiod portfolio optimization problems with decreasing time horizons.

In the classical literature on portfolio optimization, the utility function $U\left(W_{N}\right)$ is assumed to be concave to reflect aversion to risk. We consider a linear objective instead:

$$
U\left(\sum_{m=0}^{M} x_{N}^{m}\right)=\sum_{m=0}^{M} x_{N}^{m} .
$$

However, we require that the investment policy that is a solution to Problem $\left(\mathscr{P}^{r}\right)$ remain feasible for any realization of the asset returns within uncertainty sets defined by the restriction that the $D$-norm distance between the uncertain returns and their nominal values be smaller than a "robustness budget" $\Delta$. This requirement imposes an implicit risk measure in the portfolio selection process. It can be roughly interpreted as requesting insurance that the portfolio return be optimal when the total of our future estimates of the expected returns is off by up to $\Delta$.

\section{Robust formulations}

The simplest multiperiod portfolio optimization approach, the CEC, solves $\left(\mathscr{P}^{r}\right)$ by setting $\tilde{r}_{t}^{m}$ to their expected values $\check{r}_{t}^{m}$. We call such policies nominal, and study them in more detail in the computational experiments in Section 4. By contrast, the robust optimization approach treats returns as uncertain coefficients, and assumes that they vary in a pre-specified uncertainty set. It then finds a portfolio allocation strategy that remains feasible for the worst-case realizations of the uncertain returns within that uncertainty set.

Problem $\left(\mathscr{P}^{r}\right)$ contains $N \cdot M$ constraints with uncertain coefficients. Every constraint that contains uncertain coefficients adds to the dimension of the resulting robust counterpart, so we would like eliminate as many of these constraints as possible. A change in variables similar to that in Ben-Tal et al. [14] allows for reducing the number of constraints with uncertain coefficients to $N$. The trick is to work with cumulative returns $\tilde{R}_{t}^{m}, m=1, \ldots, M$, 
defined as

$$
\begin{aligned}
& R_{0}^{m}=1, \\
& \tilde{R}_{t}^{m}=\left(1+\tilde{r}_{0}^{m}\right)\left(1+\tilde{r}_{1}^{m}\right) \ldots\left(1+\tilde{r}_{t-1}^{m}\right), \quad t=1, \ldots, N .
\end{aligned}
$$

We then define

$$
\xi_{t}^{m}=\frac{x_{t}^{m}}{\tilde{R}_{t}^{m}}, \quad \eta_{t}^{m}=\frac{u_{t}^{m}}{\tilde{R}_{t}^{m}}, \quad \zeta_{t}^{m}=\frac{v_{t}^{m}}{\tilde{R}_{t}^{m}},
$$

and re-write problem $\left(\mathscr{P}^{r}\right)$ as

$$
\begin{aligned}
\left(\mathscr{P}^{R}\right) \max & w \\
\text { s.t. } \quad & w \leqslant \sum_{m=1}^{M} \tilde{R}_{N}^{m} \xi_{N}^{m}+R_{N}^{0} \xi_{N}^{0}, \\
& \xi_{t}^{m}=\xi_{t-1}^{m}-\eta_{t-1}^{m}+\zeta_{t-1}^{m}, \quad t=1, \ldots, N, \quad m=1, \ldots, M, \\
& \xi_{t}^{0}=\xi_{t-1}^{0}+\sum_{m=1}^{M}\left(1-c_{\mathrm{sell}}\right) \frac{\tilde{R}_{t-1}^{m}}{R_{t-1}^{0}} \eta_{t-1}^{m} \\
& -\sum_{m=1}^{M}\left(1+c_{\mathrm{buy}}\right) \frac{\tilde{R}_{t-1}^{m}}{R_{t-1}^{0}} \zeta_{t-1}^{m}, \quad t=1, \ldots, N, \\
& \xi_{t}^{m} \geqslant 0, \quad t=1, \ldots, N, \quad m=0, \ldots, M, \\
& \eta_{t}^{m} \geqslant 0, \quad \zeta_{t}^{m} \geqslant 0, \quad t=1, \ldots, N-1, \quad m=1, \ldots, M .
\end{aligned}
$$

Note that now only the cash account update constraints contain uncertain coefficients. We replace the equalities in these constraints by inequalities to obtain

$$
\begin{array}{ll}
\left(\mathscr{P}_{*}^{R}\right) \max & w \\
\text { s.t. } \quad & w \leqslant \sum_{m=1}^{M} \tilde{R}_{N}^{m} \xi_{N}^{m}+R_{N}^{0} \xi_{N}^{0}, \\
& \xi_{t}^{m}=\xi_{t-1}^{m}-\eta_{t-1}^{m}+\zeta_{t-1}^{m}, \quad t=1, \ldots, N, \quad m=1, \ldots, M, \\
& \xi_{t}^{0} \leqslant \xi_{t-1}^{0}+\sum_{m=1}^{M}\left(1-c_{\text {sell }}\right) \frac{\tilde{R}_{t-1}^{m}}{R_{t-1}^{0}} \eta_{t-1}^{m} \\
& -\sum_{m=1}^{M}\left(1+c_{\text {buy }}\right) \frac{\tilde{R}_{t-1}^{m}}{R_{t-1}^{0}} \zeta_{t-1}^{m}, \quad t=1, \ldots, N, \\
& \xi_{t}^{m} \geqslant 0, \quad t=1, \ldots, N, \quad m=0, \ldots, M, \\
& \eta_{t}^{m} \geqslant 0, \quad \zeta_{t}^{m} \geqslant 0, \quad t=1, \ldots, N-1, \quad m=1, \ldots, M .
\end{array}
$$

In the case of certain data, $\left(\mathscr{P}^{R}\right)$ and $\left(\mathscr{P}_{*}^{R}\right)$ are equivalent in the sense that their optimal solutions are the same. In the case of uncertain data, we need to work with $\left(\mathscr{P}_{*}^{R}\right)$ instead of $\left(\mathscr{P}^{R}\right)$, because the robust counterparts of problems with equality constraints are usually infeasible.

The simplest model of polyhedral uncertainty assumes that future cumulative returns vary in intervals $\left[\underline{R}_{t}^{m}, \bar{R}_{t}^{m}\right]$ that include their expected values $\check{R}_{t}^{m}$. The length of these intervals can be determined, for example, as a percentage of their standard deviations. The solution produced with this assumption can be viewed as a worst-case nominal policy in the sense that the program will protect against uncertainty by setting all returns to their lowest possible values-the end points of the intervals. However, this approach may be overly conservative. In practice, there is usually some kind of a correlation structure among future returns, and it rarely happens that all uncertain returns take their worst-case values 
simultaneously. It may therefore be desirable to incorporate information about the variability and the correlations of asset returns when such information is available. Furthermore, frequently more is known about the behavior of single period returns than about returns far in the future, so, for example, one can assume that the covariance matrix of the first time period returns $\Sigma_{1}$ is known. It may be reasonable also to assume that a deviation of $\tilde{R}_{t-1}^{m}$ from its nominal value could lead to an even greater deviation of $\tilde{R}_{t}^{m}$ from its nominal value. The resulting uncertainty set is

$$
P_{1}^{R}=\left\{\begin{array}{l}
\left\|\boldsymbol{\Sigma}_{1}^{-1 / 2}\left(\tilde{\mathbf{R}}_{1}-\check{\mathbf{R}}_{1}\right)\right\| \leqslant \Delta \\
\underline{\delta}_{2}^{m} \tilde{R}_{1}^{m} \leqslant \tilde{R}_{2}^{m} \leqslant \bar{\delta}_{2}^{m} \tilde{R}_{1}^{m}, m=1, \ldots, M \\
\ldots \\
\underline{\delta}_{N}^{m} \tilde{R}_{N-1}^{m} \leqslant \tilde{R}_{N}^{m} \leqslant \bar{\delta}_{N}^{m} \tilde{R}_{N-1}^{m}, m=1, \ldots, M
\end{array}\right\},
$$

where $\underline{\delta}_{t}^{m}$ and $\bar{\delta}_{t}^{m}$ are of the form $\left(1+\underline{r}_{t}^{m}\right)$ and $\left(1+\bar{r}_{t}^{m}\right)$, respectively. The main idea of this formulation is to account for the risk over the first time period, while keeping in mind some basic forecasts about the direction of asset returns in the following time periods.

If data on the covariance matrices of future cumulative returns are available, then we can impose restrictions on the movement of returns across assets

$$
P_{2}^{R}=\left\{\left\|\boldsymbol{\Sigma}_{t}^{-1 / 2}\left(\tilde{\mathbf{R}}_{t}-\check{\mathbf{R}}_{t}\right)\right\| \leqslant \Delta_{t}, t=1, \ldots, N\right\},
$$

where $\Delta_{t}$ are constants specified by the user in advance. Low values for $\Delta_{t}$ can be interpreted as a low aversion to risk. When $\Delta_{t}=0$, the investor decides based solely on expected values, and his strategy is equivalent to the nominal strategy produced by the CEC. The norm in the formulation of $P_{2}^{R}$ can be any norm. If we use the $L_{2}$ norm, we obtain Ben-Tal et al.'s [14] formulation. Note that this model does not incorporate dependencies in asset return dynamics over time explicitly; they are only present implicitly in the structure of the correlation matrices.

The robust counterparts of $\left(\mathscr{P}_{*}^{R}\right)$ with uncertainty sets $P_{1}^{R}$ or $P_{2}^{R}$, respectively, when the norm in the uncertainty sets is the D-norm, can be found using results from Bertsimas et al. [19]:

$$
\begin{array}{ll}
\max & w \\
\text { s.t. } & w \leqslant R_{N}^{0} \xi_{N}^{0}-\left(\mathbf{p}^{N}-\mathbf{q}^{N}\right)^{\prime} \mathbf{\Sigma}_{1}^{-1 / 2} \check{\mathbf{R}}_{1}-\frac{\Delta}{d} \cdot\left(\mathbf{u}^{N}\right)^{\prime} \mathbf{e}, \\
& \xi_{t}^{m}=\xi_{t-1}^{m}-\eta_{t-1}^{m}+\zeta_{t-1}^{m}, \quad t=1, \ldots, N, \quad m=1, \ldots, M, \\
& \xi_{t+1}^{0} \leqslant \xi_{t}^{0}-\left(\mathbf{p}^{t}-\mathbf{q}^{t}\right)^{\prime} \mathbf{\Sigma}_{1}^{-1 / 2} \check{\mathbf{R}}_{1}-\frac{\Delta}{d} \cdot\left(\mathbf{u}^{t}\right)^{\prime} \mathbf{e}, \quad t=1, \ldots, N-1, \\
& \xi_{1}^{0} \leqslant \xi_{0}^{0}+\sum_{m=1}^{M}\left(1-c_{\text {sell }}\right) \eta_{0}^{m}-\sum_{m=1}^{M}\left(1+c_{\text {buy }}\right) \zeta_{0}^{m} \\
& \left(\mathbf{p}^{1}-\mathbf{q}^{1}\right)^{\prime} \mathbf{\Sigma}_{1}^{-1 / 2}+\left(\begin{array}{l}
\underline{\delta}_{2}^{1}(1) \alpha_{2}^{1}(1)-\bar{\delta}_{2}^{1}(1) \beta_{2}^{1}(1) \\
\ldots \\
\underline{\delta}_{2}^{M}(1) \alpha_{2}^{M}(1)-\bar{\delta}_{2}^{M}(1) \beta_{2}^{M}(1)
\end{array}\right)^{\prime}=\left(\begin{array}{l}
-\frac{\left(1-c_{\text {sell }}\right)}{R_{1}^{0}} \eta_{1}^{1}+\frac{\left(1+c_{\text {buy }}\right)}{R_{1}^{0}} \zeta_{1}^{1} \\
-\frac{\left(1-c_{\text {sell }}\right)}{R_{1}^{0}} \eta_{1}^{M}+\frac{\left(1+c_{\text {buy }}\right)}{R_{1}^{0}} \zeta_{1}^{M}
\end{array}\right)^{\prime}, \\
& \left(\mathbf{p}^{t}-\mathbf{q}^{t}\right)^{\prime} \mathbf{\Sigma}_{1}^{-1 / 2}+\left(\begin{array}{l}
\underline{\delta}_{2}^{1}(t) \alpha_{2}^{1}(t)-\bar{\delta}_{2}^{1}(t) \beta_{2}^{1}(t) \\
\ldots \\
\underline{\delta}_{2}^{M}(t) \alpha_{2}^{M}(t)-\bar{\delta}_{2}^{M}(t) \beta_{2}^{M}(t)
\end{array}\right)^{\prime}=\mathbf{0}^{\prime}, \quad t=2, \ldots, N,
\end{array}
$$




$$
\begin{aligned}
& -\mathbf{p}^{t}-\mathbf{q}^{t}+\mathbf{u}^{t}=\mathbf{0}, \quad t=1, \ldots, N, \\
& \left(\left(\mathbf{u}^{t}\right)^{\prime} \mathbf{e}\right) \cdot \mathbf{e} \geqslant d \cdot \mathbf{u}^{t}, \quad t=1, \ldots, N, \\
& -\alpha_{\tau}^{m}(t)+\beta_{\tau}^{m}(t)+\underline{\delta}_{\tau+1}^{m} \alpha_{\tau+1}^{m}(t)-\bar{\delta}_{\tau+1}^{m} \beta_{\tau+1}^{m}(t)=0, \\
& m=1, \ldots, M, \quad t=1, \ldots, N, \quad \tau=2, \ldots, N-1, \quad \tau \neq t, \\
& -\alpha_{t}^{m}(t)+\beta_{t}^{m}(t)+\underline{\delta}_{t+1}^{m} \alpha_{t+1}^{m}(t)-\bar{\delta}_{t+1}^{m} \beta_{t+1}^{m}(t)=-\frac{\left(1-c_{\text {sell }}\right)}{R_{t}^{0}} \eta_{t}^{m}+\frac{\left(1+c_{\text {buy }}\right)}{R_{t}^{0}} \zeta_{t}^{m}, \\
& m=1, \ldots, M, \quad t=2, \ldots, N-1, \\
& -\alpha_{N}^{m}(t)+\beta_{N}^{m}(t)=0, \quad m=1, \ldots, M, \quad t=1, \ldots, N-1, \\
& -\alpha_{N}^{m}(N)+\beta_{N}^{m}(N)=-\xi_{N}^{m}, \quad m=1, \ldots, M, \\
& \xi_{t}^{m} \geqslant 0, \quad t=1, \ldots, N, \quad m=0, \ldots, M, \\
& \eta_{t}^{m} \geqslant 0, \quad \zeta_{t}^{m} \geqslant 0, \quad t=1, \ldots, N-1, \quad m=1, \ldots, M, \\
& \alpha_{\tau}^{m}(t) \geqslant 0, \quad \beta_{\tau}^{m}(t) \geqslant 0, \quad m=1, \ldots, M, \quad \tau=2, \ldots, N, \quad t=1, \ldots, N, \\
& \mathbf{p}^{t}, \mathbf{q}^{t}, \quad \mathbf{u}^{t} \geqslant 0, \quad t=1, \ldots, N
\end{aligned}
$$

and

$$
\begin{aligned}
& \max w \\
& \text { s.t. } w \leqslant \check{\mathbf{R}}_{N}^{\prime} \boldsymbol{\xi}_{N}-\frac{\Delta_{N}}{d}\left(\mathbf{w}^{N}\right)^{\prime} \mathbf{e}, \\
& \xi_{t}^{m}=\xi_{t-1}^{m}-\eta_{t-1}^{m}+\zeta_{t-1}^{m}, \quad t=1, \ldots, N, \quad m=1, \ldots, M, \\
& \xi_{1}^{0} \leqslant \xi_{0}^{0}+\sum_{m=1}^{M}\left(1-c_{\text {sell }}\right) \eta_{0}^{m}-\sum_{m=1}^{M}\left(1+c_{\text {buy }}\right) \zeta_{0}^{m} \\
& \xi_{t+1}^{0}-\xi_{t}^{0}-\left(\begin{array}{l}
-\frac{\left(1-c_{\text {sell }}\right)}{R_{t}^{0}} \eta_{t}^{1}+\frac{\left(1+c_{\text {buy }}\right)}{R_{t}^{0}} \zeta_{t}^{1} \\
-\frac{\left(1-c_{\text {sell }}\right)}{R_{t}^{0}} \eta_{t}^{M}+\frac{\left(1+c_{\text {buy }}\right)}{R_{t}^{0}} \zeta_{t}^{M}
\end{array}\right)^{\prime} \check{\mathbf{R}}_{t}+\frac{\Delta_{t}}{d}\left(\mathbf{w}^{t}\right)^{\prime} \mathbf{e} \leqslant 0, \quad t=1, \ldots, N-1, \\
& \left(2 \mathbf{p}^{N}-\mathbf{w}^{N}\right)^{\prime}=-\xi_{N}^{\prime} \boldsymbol{\Sigma}_{N}^{1 / 2}, \\
& \left(2 \mathbf{p}^{t}-\mathbf{w}^{t}\right)^{\prime}=-\left(\begin{array}{l}
-\frac{\left(1-c_{\text {sell }}\right)}{R_{t}^{0}} \eta_{t}^{1}+\frac{\left(1+c_{\text {buy }}\right)}{R_{t}^{0}} \zeta_{t}^{1} \\
\cdots \\
-\frac{\left(1-c_{\text {sell }}\right)}{R_{t}^{0}} \eta_{t}^{M}+\frac{\left(1+c_{\text {buy }}\right)}{R_{t}^{0}} \zeta_{t}^{M}
\end{array}\right)^{\prime} \boldsymbol{\Sigma}_{t}^{1 / 2} \\
& \left(\mathbf{w}^{t}\right)^{\prime} \mathbf{e} \geqslant d \cdot \mathbf{w}^{t}, \quad t=1, \ldots, N, \\
& \mathbf{w}^{t} \geqslant \mathbf{p}^{t}, \quad t=1, \ldots, N, \\
& \mathbf{p}^{t} \geqslant \mathbf{0}, \quad t=1, \ldots, N \text {, } \\
& \xi_{t}^{m} \geqslant 0, \quad t=1, \ldots, N, \quad m=0, \ldots, M, \\
& \eta_{t}^{m} \geqslant 0, \quad \zeta_{t}^{m} \geqslant 0, \quad t=1, \ldots, N-1, \quad m=1, \ldots, M .
\end{aligned}
$$

The robust counterpart for the optimization problem with uncertainty set $P_{1}^{R}$ has $2 M N^{2}+M N-2 M+N$ variables and $3 M N^{2}+8 M N-3 M+N+2$ constraints (some further reductions in the number of constraints and variables are possible, but the order of magnitude remains the same). For a portfolio of 500 stocks optimized over six time periods 
(e.g., rebalanced monthly for half a year), the optimization problem in its current form has 38,006 variables and 76,508 constraints, which can be easily handled by state-of-the-art linear solvers. The robust counterpart for the optimization problem with uncertainty set $P_{2}^{R}$ does significantly better: it has $5 M N-2 M+N$ variables and $8 M N-2 M+2 N+1$ constraints. For a portfolio of 500 stocks optimized over 6 time periods, one has to solve a linear optimization problem with 14,006 variables and 23,016 constraints.

We study the computational performance of these robust formulations in the following section.

\section{Computational results}

We conduct a series of experiments simulating different dynamics and distributions for the asset returns over several time periods, and compare the performance of portfolio strategies resulting from the following approaches:

- Single period mean-variance (henceforth abbreviated SPMV);

- Single period robust using the $D$-norm (henceforth abbreviated SPR);

- Multiperiod robust $D$-norm with uncertainty set $P_{1}^{R}$ (henceforth abbreviated MPR1);

- Multiperiod robust $D$-norm with uncertainty set $P_{2}^{R}$ (henceforth abbreviated MPR2);

- Multiperiod nominal (henceforth abbreviated MPN).

The SPMV is the standard single period portfolio optimization approach; the optimal allocations are found by solving (1) with the additional constraint that all asset weights should be greater than or equal to zero. MPR1 and MPR2 were described in Section 3. MPR1 and MPR2 result in the same optimization problem formulation if there is only one time period; SPR is the latter single period formulation. SPR solves only for the optimal single period policy with $\left\|\mid \Sigma_{1}^{-1 / 2}\left(\tilde{\mathbf{R}}_{1}-\check{\mathbf{R}}_{1}\right)\right\| \|_{d}$ as the uncertainty set for returns one period ahead. MPN finds the optimal policy by solving problem $\mathscr{P}_{*}^{R}$ with all uncertain returns fixed at their expected values.

Our experiments are designed with the following questions in mind:

1. How do the different portfolio optimization approaches perform when single period returns are drawn from the same distribution at each time period, and when there is no noise, i.e., the simulated distributions have the same parameters as the ones used as input to the corresponding optimization problems?

2. How do the approaches perform when returns are drawn from distributions with different expected returns at every time period? Does the ability to "see ahead" help the multiperiod approaches perform better?

3. How do the approaches perform when nature does not behave the way we expect probabilistically, e.g., when returns are drawn from different distributions than the ones specified at the beginning, or when the parameters of the distributions are perturbed?

We look at both symmetric and asymmetric distributions for returns.

\subsection{Symmetric (normal) single period returns}

In the first set of experiments, we simulate single period returns from a multivariate normal distribution, and we test the MPR1, SPMV, SPR, and MPN approaches on a portfolio that consists of three stocks and a riskless asset. We do not test the MPR2 approach in these experiments, because there is no closed-form expression for the covariance matrices of cumulative returns we need to formulate the robust counterpart of the optimization problem. However, we test MPR2 in the following section.

We assume that transaction costs are $1 \%$ of the amount traded. There are five trading periods: $0-4$. The investor collects his wealth at time 5. Although the dimension of the problem is small, the example illustrates the qualitative properties of the three optimization methods.

We conduct four experiments. In Experiments 1 and 2, the upper and lower bounds $\underline{\delta}_{t}^{m}$ and $\bar{\delta}_{t}^{m}$ in $P_{2}^{R}$ are both set equal to the expected value of the corresponding single period stock return at the corresponding time period. In Experiment 1, the returns are simulated from the same multivariate normal distribution at every time period. For identically distributed multivariate normal single period returns, single period mean-variance optimization results in 
Table 1

Single period expected returns $\check{r}_{t}^{1}, \breve{r}_{t}^{2}$ and $\breve{r}_{t}^{3}$ used as inputs in Experiments 2-4

\begin{tabular}{llll}
\hline Trading period $t$ & \multicolumn{2}{l}{ Expected returns } & \\
\cline { 2 - 4 } & Stock 1 & Stock 2 & Stock 3 \\
\hline 0 & 0.080 & 0.090 & 0.120 \\
1 & 0.075 & 0.080 & 0.110 \\
2 & 0.080 & 0.100 & 0.070 \\
3 & 0.080 & 0.110 & 0.090 \\
4 & 0.080 & 0.105 & 0.110 \\
\hline
\end{tabular}

theoretically optimal policies in the sense that, in the absence of transaction costs and other market frictions, they should be preferred by any risk-averse investor and should result in the best risk-return profile over multiple as well as a single investment period [20]. In Experiments 2-4, single period returns are simulated from multivariate normal distributions with different expected values at each time period (the expected values of the single period stock returns over each trading period are listed in Table 1). Therefore, single period mean-variance optimization is no longer theoretically optimal. In Experiment 2, the upper and lower bounds in the MPR1 formulation are equal, and are set to the expected values of the single period returns. Experiment 3 is the same as Experiment 2, but the upper and lower bounds in the MPR1 formulation are set to expected values $\pm 50 \%$ of the standard deviation of the corresponding stock. The setup for Experiment 4 is the same as for Experiment 3. However, while the four portfolio optimization methods are run with the data on expected returns in Table 1, in reality at each time period returns are simulated from a multivariate normal distribution whose expected values are lower than the expected values used by the four algorithms by $50 \%$ of the standard deviation for the corresponding single period return. The idea behind the design of Experiment 4 is to test how the multiperiod robust approach performs when parameters of the distribution are misspecified.

The standard deviations of the three single period asset returns are $\sigma=[0.15,0.20,0.22]$, and their correlation matrix, which remains constant over time, is

$$
\text { Cor }=\left[\begin{array}{ccc}
1 & 0.5 & 0.7 \\
0.5 & 1 & -0.2 \\
0.7 & -0.2 & 1
\end{array}\right] .
$$

The single period riskless return is 0.025 .

The value for the robustness parameter $\Delta$ in the formulation of the SPR and MPR1 approaches is set to 0.3. The value for the mean-variance optimization parameter $\lambda$ is set to 0.531 . This results in the same expected return and approximately the same variance for the optimal portfolios resulting from mean-variance optimization and single period robust $D$-norm optimization.

In each experiment, we do the following:

1. We simulate 1000 paths of the cumulative returns of each stock over five periods. For each path:

(a) We assume that at time 0 , we hold 1 unit in the riskless asset, and 0 units in the three risky assets. We compute the optimal strategy for each of the four methods: the SPMV, the SPR, the MPR1, and the MPN.

(b) We take one step forward using these optimal strategies, and update our holdings according to the realized single period return over that time period.

(c) Given the new holdings and the data on single period expected returns for the remaining periods, we re-compute the optimal strategies for the next time period.

(d) We proceed in the same way until the last trading period, time 4. We store the realized final holdings obtained with each method.

2. We compute the descriptive statistics for the annualized returns from the 1000 scenarios for each of the four methods.

Results. The performance of the optimal strategies of the four methods is shown in Table 2. We note that the results are presented in terms of annualized returns rather than cumulative returns. The notation used is as follows: (a) Mean: 
Table 2

Performance of the SPMV, the SPR, the MPR1, and the MPN approaches in Experiments 1-4 when single period returns follow a multivariate normal distribution. Transaction costs are assumed to be $1 \%$ of the amount traded

\begin{tabular}{|c|c|c|c|c|c|c|c|}
\hline Exp. no. & Method & Mean & StdDev & Min & Max & Ratio & Prob $(\%)$ \\
\hline \multirow[t]{4}{*}{1} & SPMV & 0.0985 & 0.0674 & -0.0982 & 0.3204 & 1.46 & 7.40 \\
\hline & SPR & 0.0978 & 0.0676 & -0.1052 & 0.3227 & 1.45 & 7.50 \\
\hline & MPR1 & 0.0986 & 0.0506 & -0.0081 & 0.3255 & 1.95 & 0.10 \\
\hline & MPN & 0.0972 & 0.0962 & -0.1855 & 0.4105 & 1.01 & 15.90 \\
\hline \multirow[t]{4}{*}{2} & SPMV & 0.0892 & 0.0667 & -0.1241 & 0.2987 & 1.34 & 9.30 \\
\hline & SPR & 0.0883 & 0.0694 & -0.1488 & 0.2989 & 1.27 & 9.80 \\
\hline & MPR1 & 0.0905 & 0.0387 & 0.0170 & 0.2657 & 2.34 & 0.00 \\
\hline & MPN & 0.0831 & 0.0949 & -0.2280 & 0.3780 & 0.88 & 19.10 \\
\hline \multirow[t]{4}{*}{3} & SPMV & 0.0876 & 0.0664 & -0.1454 & 0.3113 & 1.32 & 8.90 \\
\hline & SPR & 0.0876 & 0.0694 & -0.1675 & 0.2899 & 1.26 & 9.40 \\
\hline & MPR1 & 0.0902 & 0.0370 & 0.0211 & 0.2415 & 2.44 & 0.00 \\
\hline & MPN & 0.0852 & 0.0971 & -0.3109 & 0.3569 & 0.88 & 18.40 \\
\hline \multirow[t]{4}{*}{4} & SPMV & 0.0781 & 0.0670 & -0.1242 & 0.3202 & 1.17 & 11.80 \\
\hline & SPR & 0.0775 & 0.0693 & -0.1299 & 0.3006 & 1.12 & 13.40 \\
\hline & MPR1 & 0.0876 & 0.0393 & 0.0176 & 0.2892 & 2.23 & 0.00 \\
\hline & MPN & 0.0720 & 0.0951 & -0.2177 & 0.3496 & 0.76 & 22.30 \\
\hline
\end{tabular}

average portfolio return resulting from each method over 1000 simulated stock return paths; (b) StdDev: standard deviation of portfolio return over the 1000 paths; (c) Min (Max): minimum (maximum) realized portfolio return over the 1000 paths; (d) Ratio: ratio of realized portfolio mean to realized portfolio standard deviation; (e) Prob: empirical probability of loss, i.e., the probability that the annualized realized return is less than 0 .

It can be observed from the computational results in Table 2 that the MPR1 approach achieves better average return, probability of loss, and mean-to-standard deviation ratio than the other methods. Its dominance in the mean-to-standard deviation ratio is particularly important, because it shows that the risk of the portfolio is decreased at no cost to the expected portfolio return. Standard deviation is appropriate as a measure of risk in these experiments, because the return distributions are symmetric (normal).

The MPR1 approach also has better worst-case scenario performance than the other approaches. It is interesting to note the difference between MPR1's worst-case performance in Experiments 2 and 3. In Experiment 3, where the upper and lower bounds on future stock returns are set to be $50 \%$ of the corresponding returns' standard deviations away from the returns' expected values (as opposed to expected values, as in Experiment 2), the MPR1 approach becomes more conservative, and its worst-case scenario performance improves. Its mean-to-standard deviation ratio improves as well. The conservativeness of the MPR1 approach can therefore be controlled by the width of the bounds on future returns. This observation is confirmed also by the experiments with asymmetric distributions in the next section.

The value selected for the $D$-norm in the MPR1 formulation makes a difference. Table 3 shows simulation results for Experiment 2 when the $D$-norm is set to 1 or to the number of stocks in the portfolio, 3 . It appears that larger values for the $D$-norm improve the mean-to-standard deviation ratio; however, the worst-case performance suffers slightly.

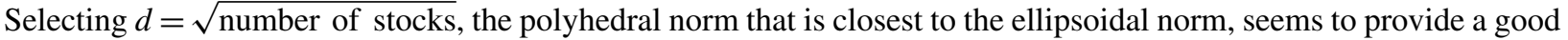
balance.

\subsection{Asymmetric (lognormal) single period returns}

The next series of experiments study the performance of the robust approaches when returns follow skewed distributions. We consider a portfolio of 25 stocks over five time periods, and use the simulation setup of Ben-Tal et al. [14]: the stochastic model of the data is a simple factor model

$$
\begin{aligned}
& \ln \left(1+r_{t}^{m}\right)=\mathbf{\Omega}_{m}^{\prime}\left[\kappa \cdot \mathbf{e}+\sigma \cdot \boldsymbol{v}_{t}\right], \quad t=0,1, \ldots, N-1, \quad m=1, \ldots, M, \\
& \ln \left(1+r_{t}^{0}\right)=\kappa, \quad t=0,1, \ldots, N-1,
\end{aligned}
$$


Table 3

Performance of the SPMV, the SPR, the MPR1, and the MPN approaches for different values of the $D$-norm in Experiment 2 when single period returns follow a multivariate normal distribution. Transaction costs are assumed to be $1 \%$ of the amount traded

\begin{tabular}{|c|c|c|c|c|c|c|c|}
\hline$D$-norm & Method & Mean & StdDev & Min & Max & Ratio & Prob $(\%)$ \\
\hline \multirow[t]{4}{*}{1} & SPMV & 0.0869 & 0.0726 & -0.1809 & 0.3255 & 1.20 & 11.40 \\
\hline & SPR & 0.0758 & 0.0710 & -0.1701 & 0.3005 & 1.07 & 14.80 \\
\hline & MPR1 & 0.0897 & 0.0407 & 0.0238 & 0.2792 & 2.21 & 0.00 \\
\hline & MPN & 0.0843 & 0.0967 & -0.2630 & 0.3351 & 0.87 & 20.00 \\
\hline \multirow[t]{4}{*}{3} & SPMV & 0.0775 & 0.0480 & -0.0819 & 0.1901 & 1.61 & 5.60 \\
\hline & SPR & 0.0831 & 0.0575 & -0.0974 & 0.2461 & 1.44 & 6.40 \\
\hline & MPR1 & 0.0903 & 0.0360 & 0.0159 & 0.2534 & 2.51 & 0.00 \\
\hline & MPN & 0.0795 & 0.0913 & -0.2274 & 0.3576 & 0.87 & 19.40 \\
\hline
\end{tabular}

where $\left\{\boldsymbol{v}_{0}, \boldsymbol{v}_{1}, \ldots, \boldsymbol{v}_{N-1}\right\}$ are independent $K$-dimensional Gaussian random vectors with zero mean and unit covariance matrix; $\mathbf{e} \in \mathfrak{R}^{K}=(1, \ldots, 1)^{\prime} ; \boldsymbol{\Omega}_{m} \in \mathfrak{R}_{+}^{K}$ are fixed vectors; and $\kappa, \sigma>0$ are fixed reals. Single period returns are therefore lognormal. The assumptions allow for computing the expected values and the covariances of the cumulative returns at time $t$ in closed form. All simulation parameters are selected as in Ben-Tal et al. [14].

We conduct the following experiments:

Experiment 1 . The single period returns for each asset are assumed to be independent and identically distributed across time periods. We expect that in this case single period portfolio optimization should do about the same as multiperiod models, because having the ability to "see" far in the future has no benefit. However, it is possible that the presence of transaction costs will affect the anticipated outcome. For every time period a vector of factor realizations $\boldsymbol{v}_{t}$ is drawn from a multivariate normal distribution with mean $\mathbf{0}$ and unit covariance matrix, and the realized returns are computed using (5). The upper and lower bounds in MPR1 are set to the expected values of the corresponding single period returns.

Experiment 2 is the same as Experiment 1, but the simulated returns are perturbed: after a realization of the vector $\boldsymbol{v}_{t}$ is obtained and the returns are computed using (5), $10 \%$ of the value of each realized return is subtracted. The simulated values are therefore lower on average than the optimization problems "expect."

Experiment 3 is the same as Experiment 2, but the upper and lower bounds for each asset in the MPR1 formulation are set to be $50 \%$ of the standard deviation of the corresponding asset.

In Experiment 4, the single period expected returns for the first time period are the same as the expected returns in Experiments 1-3, but the expected single period returns in later time periods are different. The MPR1 and the MPR2 "know" the expected returns more than one time period ahead. The upper and lower bounds for each asset in the MPR1 formulation are set to the expected values of the single period returns.

Experiment 5 is the same as Experiment 4; however, $10 \%$ of the realized returns is subtracted in all simulations. The expected values for returns used as inputs in all optimization models, as well as the covariance matrices used in the formulation of MPR2, are therefore not correct, so the models are misspecified. The upper and lower bounds for each asset return in the MPR1 formulation are set to 50\% of the standard deviation of the single period returns. The results of Experiment 5 are particularly important, because the setting of the experiment is the most realistic one.

Experiment 6 is the same as Experiment 1, but single period returns are drawn from a multivariate normal distribution (instead of a lognormal distribution) with the same expected value and single period covariance matrix as the single period lognormal distribution for returns.

The experiments are run for values of the $d$-norm equal to $\sqrt{\text { number of stocks }}$ and $d=$ number of stocks, and the values of $\lambda$ and $\Delta$ change correspondingly. There are five time periods and 1000 simulations per experiment, as in the previous section. We start with 1 dollar in the riskless asset, and 0 dollars in the risky assets. Standard deviations and means are not as informative as they were in the previous section, because the distributions in the simulations are skewed. We still provide the values for mean, standard deviation, and their ratio, but we also provide information about the 5th percentile, the 50th percentile (the median), and the 95th percentile of the final distribution of portfolio returns. The results are presented in Tables 4 and 5. Table 6 contains pairwise comparisons of the realized returns with each of the five approaches for $d=\sqrt{\text { number of stocks }}$ and $d=$ number of stocks. 
Table 4

Performance of the SPMV, the SPR, the MPR1, the MPR2, and the MPN approaches when the $D$-norm equals the square root of the number of stocks in the portfolio in Experiments 1-6. The market risk index equals 0.3704 . The values of the parameters in the optimization problems are $\Delta=0.1, \lambda=0.2$. Transaction costs are assumed to be $1 \%$ of the amount traded

\begin{tabular}{|c|c|c|c|c|c|c|c|c|c|c|}
\hline Exp. no. & Method & Mean & StdDev & Ratio & Min & 5th Per & 50th Per & 95th Per & $\operatorname{Max}$ & Prob $(\%)$ \\
\hline \multirow[t]{5}{*}{1} & SPMV & 0.1933 & 0.1396 & 1.39 & -0.1983 & -0.0255 & 0.1798 & 0.4283 & 0.7441 & 7.50 \\
\hline & SPR & 0.1935 & 0.1535 & 1.26 & -0.2285 & -0.0387 & 0.1793 & 0.4637 & 0.8517 & 8.40 \\
\hline & MPR1 & 0.2875 & 0.2338 & 1.23 & -0.1866 & -0.0130 & 0.2458 & 0.7497 & 1.4013 & 6.70 \\
\hline & MPR2 & 0.3468 & 0.2598 & 1.34 & -0.1135 & 0.0100 & 0.2964 & 0.8543 & 1.4306 & 4.70 \\
\hline & MPN & 0.1805 & 0.1793 & 1.01 & -0.2507 & -0.0834 & 0.1660 & 0.5021 & 0.9159 & 15.90 \\
\hline \multirow[t]{5}{*}{2} & SPMV & 0.0715 & 0.1252 & 0.57 & -0.2199 & -0.1176 & 0.0632 & 0.2951 & 0.5728 & 30.10 \\
\hline & SPR & 0.0727 & 0.1359 & 0.53 & -0.2689 & -0.1289 & 0.0600 & 0.3152 & 0.6669 & 32.20 \\
\hline & MPR1 & 0.1455 & 0.1971 & 0.74 & -0.2386 & -0.1121 & 0.1117 & 0.5098 & 1.3134 & 21.90 \\
\hline & MPR2 & 0.1844 & 0.2058 & 0.90 & -0.2320 & -0.0889 & 0.1496 & 0.5493 & 1.0864 & 18.40 \\
\hline & MPN & 0.0617 & 0.1598 & 0.39 & -0.3128 & -0.1739 & 0.0468 & 0.3491 & 0.6539 & 39.20 \\
\hline \multirow[t]{5}{*}{3} & SPMV & 0.0709 & 0.1219 & 0.58 & -0.2915 & -0.1201 & 0.0657 & 0.2724 & 0.5364 & 29.90 \\
\hline & SPR & 0.0702 & 0.1321 & 0.53 & -0.2870 & -0.1301 & 0.0634 & 0.3001 & 0.6871 & 31.20 \\
\hline & MPR1 & 0.1357 & 0.1763 & 0.77 & -0.2093 & -0.0996 & 0.1102 & 0.4688 & 0.9867 & 22.60 \\
\hline & MPR2 & 0.1774 & 0.2019 & 0.88 & -0.2021 & -0.0905 & 0.1494 & 0.5380 & 1.5102 & 17.60 \\
\hline & MPN & 0.0553 & 0.1562 & 0.35 & -0.3413 & -0.1691 & 0.0435 & 0.3371 & 0.6691 & 38.40 \\
\hline \multirow[t]{5}{*}{4} & SPMV & 0.1968 & 0.1623 & 1.21 & -0.3010 & -0.0647 & 0.1948 & 0.4738 & 0.6814 & 11.20 \\
\hline & SPR & 0.1869 & 0.1839 & 1.02 & -0.3858 & -0.0973 & 0.1791 & 0.5112 & 0.7703 & 15.10 \\
\hline & MPR1 & 0.6941 & 0.4544 & 1.53 & -0.2022 & 0.0899 & 0.6129 & 1.5331 & 2.7689 & 1.90 \\
\hline & MPR2 & 0.2872 & 0.2014 & 1.43 & -0.1629 & 0.0009 & 0.2577 & 0.6504 & 1.5458 & 4.80 \\
\hline & $\mathrm{MPN}$ & 0.1642 & 0.2069 & 0.79 & -0.3493 & -0.1578 & 0.1557 & 0.5184 & 0.9441 & 22.40 \\
\hline \multirow[t]{5}{*}{5} & SPMV & 0.0733 & 0.1552 & 0.47 & -0.3266 & -0.1579 & 0.0588 & 0.3403 & 0.8522 & 33.20 \\
\hline & SPR & 0.0623 & 0.1703 & 0.37 & -0.3687 & -0.1903 & 0.0460 & 0.3635 & 0.8555 & 38.90 \\
\hline & MPR1 & 0.4212 & 0.3794 & 1.11 & -0.2355 & -0.0158 & 0.3404 & 1.0652 & 2.9457 & 6.60 \\
\hline & MPR2 & 0.1426 & 0.1818 & 0.78 & -0.2280 & -0.0973 & 0.1151 & 0.4712 & 1.2849 & 21.20 \\
\hline & $\mathrm{MPN}$ & 0.0502 & 0.1950 & 0.26 & -0.4059 & -0.2256 & 0.0306 & 0.3878 & 1.0548 & 43.70 \\
\hline \multirow[t]{5}{*}{6} & SPMV & 0.2312 & 0.1376 & 1.68 & -0.2354 & -0.0046 & 0.2369 & 0.4454 & 0.5953 & 5.40 \\
\hline & SPR & 0.2278 & 0.1831 & 1.24 & -0.3578 & -0.0721 & 0.2324 & 0.5214 & 0.7698 & 11.50 \\
\hline & MPR1 & 0.3239 & 0.2934 & 1.10 & -0.6136 & -0.1254 & 0.3054 & 0.8267 & 1.3822 & 12.50 \\
\hline & MPR2 & 0.4399 & 0.2956 & 1.49 & -0.3519 & -0.0042 & 0.4140 & 0.9790 & 1.5433 & 5.10 \\
\hline & MPN & 0.1743 & 0.2794 & 0.62 & -0.7380 & -0.3052 & 0.1796 & 0.6384 & 1.1475 & 26.40 \\
\hline
\end{tabular}

Results. The computational results for Experiment 1 for both values of the $D$-norm show that when returns are identically distributed in all time periods, the ability of multiperiod models to take into consideration information about events further in the future is not too valuable when it comes to mean-to-standard deviation ratio. However, MPR1's and MPR2's realized average returns are higher than the realized average returns of the single period models, their worst-case scenarios are better, and so are their best case scenarios, probability of loss, and 50th percentiles. MPR1 and MPR2 also dominate in the comparisons on a scenario-by-scenario basis (Table 6).

The dominance of the multiperiod approaches becomes stronger when single period returns have different expected values in the different trading periods, as illustrated by the results from Experiment 4 . The robust multiperiod approaches also perform better than the single period approaches in terms of realized average return and mean-to-standard deviation ratio, worst-case and median performance, and probability of loss when parameters in the models are misspecified (Experiments 2, 3, and 5).

When returns are simulated from a normal (instead of lognormal) distribution and are identically distributed in all time periods (Experiment 6), the SMPV approach has a very good worst-case performance and mean-to-standard deviation ratio relative to the other approaches (the ratio is relevant in Experiment 6, because symmetric distributions are involved). This is to be expected. We note, however, that the MPR2 approach does very well too, despite the fact that the values for the covariances in the MPR2 formulation are no longer correct. 
Table 5

Performance of the SPMV, the SPR, the MPR1, the MPR2, and the MPN approaches for $D$-norm equal to the number of stocks in the portfolio in Experiments 1-6. The market risk index equals 0.3704 . The values of the parameters in the optimization problems are $\Delta=0.1, \lambda=0.9$. Transaction costs are assumed to be $1 \%$ of the amount traded

\begin{tabular}{|c|c|c|c|c|c|c|c|c|c|c|}
\hline Exp. no. & Method & Mean & StdDev & Ratio & Min & 5th Per & 50th Per & 95th Per & Max & Prob (\%) \\
\hline \multirow[t]{5}{*}{1} & SPMV & 0.1920 & 0.1524 & 1.26 & -0.2110 & -0.0519 & 0.1854 & 0.4630 & 0.6714 & 10.60 \\
\hline & SPR & 0.1926 & 0.1544 & 1.25 & -0.1962 & -0.0539 & 0.1839 & 0.4612 & 0.7134 & 10.50 \\
\hline & MPR1 & 0.2879 & 0.2374 & 1.21 & -0.2595 & -0.0379 & 0.2461 & 0.7501 & 1.4198 & 8.10 \\
\hline & MPR2 & 0.3654 & 0.2594 & 1.41 & -0.1416 & 0.0214 & 0.3242 & 0.8496 & 1.3916 & 4.20 \\
\hline & MPN & 0.1818 & 0.1830 & 0.99 & -0.3015 & -0.1023 & 0.1755 & 0.5020 & 0.8276 & 15.50 \\
\hline \multirow[t]{5}{*}{2} & SPMV & 0.0693 & 0.1412 & 0.49 & -0.3227 & -0.1509 & 0.0614 & 0.3069 & 0.6111 & 32.70 \\
\hline & SPR & 0.0694 & 0.1418 & 0.49 & -0.3174 & -0.1546 & 0.0606 & 0.3125 & 0.6238 & 31.90 \\
\hline & MPR1 & 0.1353 & 0.2014 & 0.67 & -0.2978 & -0.1286 & 0.1027 & 0.5147 & 1.2264 & 25.90 \\
\hline & MPR2 & 0.1985 & 0.2215 & 0.90 & -0.2629 & -0.0959 & 0.1601 & 0.6127 & 1.2288 & 16.80 \\
\hline & MPN & 0.0535 & 0.1668 & 0.32 & -0.3578 & -0.1961 & 0.0375 & 0.3469 & 0.7365 & 40.20 \\
\hline \multirow[t]{5}{*}{3} & SPMV & 0.0795 & 0.1320 & 0.60 & -0.2463 & -0.1174 & 0.0717 & 0.3003 & 0.6016 & 28.80 \\
\hline & SPR & 0.0794 & 0.1321 & 0.60 & -0.2551 & -0.1217 & 0.0706 & 0.3013 & 0.6301 & 28.90 \\
\hline & MPR1 & 0.1550 & 0.1725 & 0.90 & -0.2296 & -0.0832 & 0.1323 & 0.4609 & 1.0483 & 19.00 \\
\hline & MPR2 & 0.2093 & 0.2074 & 1.01 & -0.2196 & -0.0730 & 0.1807 & 0.5920 & 0.9824 & 15.20 \\
\hline & MPN & 0.0713 & 0.1593 & 0.45 & -0.3762 & -0.1682 & 0.0527 & 0.3446 & 0.6660 & 36.50 \\
\hline \multirow[t]{5}{*}{4} & SPMV & 0.1952 & 0.1799 & 1.08 & -0.3166 & -0.0709 & 0.1841 & 0.5092 & 1.0402 & 14.60 \\
\hline & SPR & 0.1975 & 0.1795 & 1.10 & -0.2788 & -0.0617 & 0.1793 & 0.5137 & 0.9959 & 14.00 \\
\hline & MPR1 & 0.7355 & 0.4904 & 1.50 & -0.0935 & 0.0925 & 0.6630 & 1.6734 & 3.4678 & 1.00 \\
\hline & MPR2 & 0.2976 & 0.2061 & 1.44 & -0.1931 & 0.0095 & 0.2729 & 0.6878 & 1.5249 & 4.30 \\
\hline & MPN & 0.1705 & 0.2056 & 0.83 & -0.2994 & -0.1464 & 0.1596 & 0.5384 & 0.9891 & 19.80 \\
\hline \multirow[t]{5}{*}{5} & SPMV & 0.0823 & 0.1789 & 0.46 & -0.4397 & -0.1827 & 0.0641 & 0.4222 & 0.7995 & 33.40 \\
\hline & SPR & 0.0834 & 0.1754 & 0.48 & -0.3711 & -0.1796 & 0.0681 & 0.4131 & 0.7997 & 33.70 \\
\hline & MPR1 & 0.4123 & 0.3630 & 1.14 & -0.2324 & -0.0401 & 0.3531 & 1.1013 & 2.4837 & 7.40 \\
\hline & MPR2 & 0.1507 & 0.1794 & 0.84 & -0.1955 & -0.1001 & 0.1317 & 0.4840 & 1.2104 & 19.80 \\
\hline & MPN & 0.0617 & 0.1959 & 0.31 & -0.3731 & -0.2353 & 0.0431 & 0.4121 & 0.9282 & 38.90 \\
\hline \multirow[t]{5}{*}{6} & SPMV & 0.2274 & 0.1735 & 1.31 & -0.4397 & -0.0588 & 0.2415 & 0.5054 & 0.6427 & 9.80 \\
\hline & SPR & 0.2248 & 0.1806 & 1.24 & -0.4204 & -0.0515 & 0.2230 & 0.5299 & 0.6928 & 10.30 \\
\hline & MPR1 & 0.3077 & 0.2898 & 1.06 & -0.5451 & -0.1330 & 0.2917 & 0.8462 & 1.4747 & 14.00 \\
\hline & MPR2 & 0.4942 & 0.3163 & 1.56 & -0.3733 & 0.0307 & 0.4621 & 1.0778 & 1.4974 & 3.40 \\
\hline & MPN & 0.1627 & 0.2818 & 0.58 & -0.6515 & -0.2803 & 0.1638 & 0.5942 & 1.0146 & 27.80 \\
\hline
\end{tabular}

While MPR1 and MPR2 both have excellent performance in all experiments, it is worth noting that MPR2 tends to do better than MPR1 in terms of realized average return, worst-case return, and probability of loss when returns are identically distributed in all time periods (as is the case in Experiments 1-3, and 6). MPR1, on the other hand, performs extremely well when returns are not identically distributed, or when parameters in the model are misspecified, as is the case in Experiments 4 and 5. Moreover, it appears that its worst-case performance can be improved by widening the bounds on returns in the formulation. This can be seen by comparing MPR1's worst-case performance in Experiments 2 and 3.

Experiments 1-6 were run for markets with different risk indices. We used a measure of "riskiness" suggested by BenTal et al. [14]: the maximum probability of loss over all asset returns in the first time period, $\max _{m=1, \ldots, M} \operatorname{Pr}\left(r_{0}^{m}<0\right)$. When this probability is higher, the market it more risky (the risk index for the data set used for the results in Tables 4-6 is 0.3704). We omit the results for the sake of brevity. The riskiness of the market, or the choice of norm $(d=\sqrt{\text { number of stocks }}$ or $d=$ number of stocks) do not change our overall conclusions.

\section{Concluding remarks}

We suggested different robust formulations of the multiperiod portfolio management problem with transaction costs, and showed, via simulations, that robust polyhedral optimization in particular can enhance the performance of single 
Table 6

Pairwise comparisons of the performance of the SPMV, the SPR, the MPR1, the MPR2, and the MPN approaches in Experiments 1-6 for different values of the $D$-norm. The market risk index equals 0.3704 . The value in each cell indicates the percentage of times the "row" policy resulted in better final portfolio return than the "column" policy

\begin{tabular}{|c|c|c|c|c|c|c|c|c|c|c|}
\hline & Experim & $1, d=\checkmark$ & mber of & & & Experin & $1, d=$ & ber of $s t$ & & \\
\hline & SPMV & SPR & MPR1 & MPR2 & MPN & SPMV & SPR & MPR1 & MPR2 & MPN \\
\hline SPMV & & 47.4 & 28.4 & 12.5 & 52.1 & & 42.5 & 25.4 & 2.7 & 51.4 \\
\hline SPR & 52.6 & & 26.6 & 6.9 & 54.3 & 57.5 & & 27.3 & 2.6 & 54.5 \\
\hline MPR1 & 71.6 & 73.4 & & 37.6 & 79.4 & 74.6 & 72.7 & & 36.5 & 79.6 \\
\hline MPR2 & 87.5 & 93.1 & 62.4 & & 94.1 & 97.3 & 97.4 & 63.5 & & 90.0 \\
\hline & Experim & $2, d=\checkmark$ & mber of & & & Experin & $2, d=$ & ber of $s t$ & & \\
\hline SPMV & & 45.0 & 28.9 & 12.6 & 52.5 & & 41.3 & 29.1 & 5.5 & 53.5 \\
\hline SPR & 55.0 & & 26.7 & 6.5 & 54.1 & 58.7 & & 29.7 & 5.5 & 57.2 \\
\hline MPR1 & 71.1 & 73.3 & & 37.6 & 79.7 & 70.9 & 70.3 & & 33.5 & 80.1 \\
\hline MPR2 & 87.4 & 93.5 & 62.4 & & 91.2 & 94.5 & 94.5 & 66.5 & & 87.4 \\
\hline & Experim & $3, d=\checkmark$ & mber of & & & Experin & $3, d=$ & ber of $s t$ & & \\
\hline SPMV & & 44.4 & 27.4 & 13.9 & 55.9 & & 42.5 & 9.5 & 4.5 & 47.8 \\
\hline SPR & 55.6 & & 24.9 & 7.7 & 58.0 & 57.5 & & 10.6 & 5.0 & 51.7 \\
\hline MPR1 & 72.6 & 75.1 & & 31.4 & 83.1 & 90.5 & 89.4 & & 31.8 & 83.9 \\
\hline MPR2 & 86.1 & 92.3 & 68.6 & & 93.5 & 95.5 & 95.0 & 68.2 & & 86.9 \\
\hline & Experim & $4, d=\checkmark$ & mber of & & & Experin & $4, d=$ & ber of $s t$ & & \\
\hline SPMV & & 58.7 & 1.2 & 31.9 & 56.9 & & 50.1 & 0.0 & 31.2 & 54.9 \\
\hline SPR & 41.3 & & 0.5 & 31.4 & 53.5 & 49.9 & & 0.1 & 31.6 & 55.7 \\
\hline MPR1 & 98.8 & 99.5 & & 88.1 & 96.9 & 100.0 & 99.9 & & 88.0 & 96.0 \\
\hline MPR2 & 68.1 & 68.6 & 11.9 & & 73.7 & 68.8 & 68.4 & 12.0 & & 71.7 \\
\hline & Experim & $5, d=\checkmark$ & mber of & & & Experin & $5, d=$ & ber of $s t$ & & \\
\hline SPMV & & 59.4 & 0.9 & 35.0 & 56.3 & & 52.9 & 0.0 & 33.8 & 55.6 \\
\hline SPR & 40.6 & & 0.0 & 32.7 & 53.5 & 47.1 & & 0.2 & 35.1 & 56.0 \\
\hline MPR1 & 99.1 & 100.0 & & 85.3 & 93.2 & 100.0 & 99.8 & & 82.9 & 91.2 \\
\hline MPR2 & 65.0 & 67.3 & 14.7 & & 71.6 & 66.2 & 64.9 & 17.1 & & 67.7 \\
\hline & Experim & $6, d=\checkmark$ & mber of & & & Experin & $6, d=$ & ber of $s t$ & & \\
\hline SPMV & & 48.6 & 35.6 & 16.6 & 59.7 & & 46.9 & 34.4 & 7.0 & 58.2 \\
\hline SPR & 51.4 & & 31.8 & 9.1 & 58.9 & 53.1 & & 35.0 & 6.4 & 62.2 \\
\hline MPR1 & 64.4 & 68.2 & & 30.0 & 81.3 & 65.6 & 65.0 & & 25.4 & 81.4 \\
\hline MPR2 & 83.4 & 90.9 & 70.0 & & 93.7 & 93.0 & 93.6 & 74.6 & & 92.6 \\
\hline
\end{tabular}

period and deterministic multiperiod portfolio optimization methods. Robust polyhedral optimization models avoid the curse of dimensionality and can be solved with commercially available linear programming software, yet they allow for flexible formulations in which the anticipation of future expected returns, as well as a tolerance level for the error in our forecasts, can be explicitly modeled. We focused our attention on two multiperiod formulations in particular: the MPR2 approach, which is similar to the approach suggested by Ben-Tal et al. [14], and the MPR1 approach. The MPR2 approach requires solving optimization problems of smaller dimension than the MPR1 approach, and tends to outperform the single period mean-variance approach and sometimes the MPR1 approach when the parameters in the simulation model are correctly specified. This is because the MPR2 formulation incorporates more information about variability and direction of movement of future asset returns than the single period mean-variance or the MPR1 approach. When such information is available, and is believed to be reasonably accurate, MPR2 may be preferable to use. However, the MPR1 approach appears to be the most robust with respect to misspecifications in the parameters of the model, and clearly dominates the other approaches in situations that are most similar to real life. Moreover, 
its worst-case behavior can be controlled by varying the width of the bounds on the predictions for future portfolio returns.

In conclusion, robust multiperiod portfolio approaches show excellent potential as alternatives to classical single period portfolio optimization models, independently of the shape (symmetric or skewed) of asset return distributions.

\section{Acknowledgment}

The second author is grateful for financial support from the Babson College Board of Research.

\section{References}

[1] Markowitz HM. Portfolio selection. Journal of Finance 1952;7:77-91.

[2] Markowitz HM. Portfolio selection: efficient diversification of investments. 2nd ed., Cambridge, MA: Basil Blackwell; 1991.

[3] Merton R. Optimum consumption and portfolio rules in a continuous time model. Journal of Economic Theory 1971;3:373-413.

[4] Merton R. Continuous-time finance. Cambridge, MA: Blackwell Publishers; 1990.

[5] Samuelson PA. Lifetime portfolio selection by dynamic stochastic programming. Review of Economics and Statistics 1969;51:239-46.

[6] Cox J, Huang CF. Optimal consumption and portfolio choices when asset prices follow a diffusion process. Journal of Economic Theory 1989;49:33-83.

[7] Chryssikou E. Multiperiod portfolio optimization in the presence of transaction costs. PhD thesis, MIT, Cambridge, MA; 1998.

[8] Mulvey JM, Vladimirou H. Stochastic network optimization models for investment planning. Annals of Operations Research 1989;20: $187-217$.

[9] Dantzig GB, Infanger G. Multi-stage stochastic linear programs for portfolio optimization. Annals of Operations Research 1993;45:59-76.

[10] Carino DR, Turner AL. Multiperiod asset allocation with derivative assets. In: Ziemba WT, Mulvey JM, editors. Worldwide asset and liability modeling. Cambridge: Cambridge University Press; 1998.

[11] Steinbach MC. Markowitz revisited single-period and multi-period mean-variance models. SIAM Review 2001;43:31-85.

[12] Ben-Tal A, Nemirovski A. Robust convex optimization. Mathematics of Operations Research 1998;23:769-805.

[13] El Ghaoui L, Lebret H. Robust solutions to least-squares problems with uncertain data. SIAM Journal of Matrix Analysis Applications 1997; 18:1035-64.

[14] Ben-Tal A, Margelit T, Nemirovski A. Robust modeling of multi-stage portfolio problems. In: Frenk H, Roos K, Terlaky T, Zhang S, editors. High-performance optimization. Dordrecht: Kluwer Academic Publishers; 2000. p. 303-28.

[15] Bertsekas DP. Dynamic programming and optimal control. Belmont, MA, USA: Athena Scientific; 1995.

[16] Ben-Tal A, Nemirovski A. Robust solutions of uncertain linear programs. Operations Research Letters 1999;25:1-13.

[17] Bertsimas D, Darnell C, Soucy R. Portfolio construction through mixed-integer programming at Grantham, Mayo, Van Otterloo and Company. Interfaces 1999;29:49-66.

[18] Bertsimas D, Sim M. Robust discrete optimization and network flows. Mathematical Programming Series B 2003;98:49-71.

[19] Bertsimas D, Pachamanova D, Sim M. Robust linear optimization under general norms. Operations Research Letters 2004;32:510-6.

[20] Chamberlain G. A characterization of the distributions that imply mean-variance utility functions. Journal of Economic Theory 1983;29: $185-201$. 